\title{
The Emergence of Specialty Pharmacy
}

\author{
by Gordon J. Vanscoy
}

$\mathrm{F}$ or all that has been written about managed care, it's ironic that more has not been written about specialty pharmacy, particularly since specialized pharmaceutical care has significant potential for managing such catastrophic illnesses as human immunodeficiency virus (HIV) infection/acquired immunodeficiency syndrome (AIDS), and cancer. One reason specialty pharmaceutical care may not have been on the radar screen of the managed care industry is that the aggregate costs of managing each catastrophic illness population is small in comparison to more common afflictions such as asthma or diabetes. In addition, mainstream disease management models and actuarials do not exist for specialized disease states. Finally, the managed care industry has been slow to recognize the value of managing patients with rare yet catastrophic conditions. What role could pharmacy play?

Specialty pharmacy has been growing quietly since the 1980s. In the mid1980s, when some of the country's first solid organ transplants took place in Pittsburgh, recipients began to rely on a local corner drugstore for their pharmaceutical care. Stadtlanders Pharmacy emerged with the specialty expertise and pharmaceuticals these patients required, including assistance with medication reimbursement, counseling, and education. Stadtlanders now provides nation- wide care to more than 55,000 patients who are living with organ transplants, HIV/AIDS, serious mental illness, cancer, or hepatitis C. Several companies currently provide specialty pharmacy services, among them Stadtlanders, CVS Procare (HIV/AIDS), Chronimed (organ transplant recipients), and Priority Healthcare (oncology, HIV/AIDS, and hepatitis).

\section{The Costs of Catastrophic IIIness}

There are many ways to define calastrophic illness. However, a simple way to identify them is to analyze patients managed by an insurer's catastrophic case managers, including organ transplant, HIV/AIDS, oncology, and hepatitis C patients.

Although catastrophic illnesses may be relatively rare, they are very costly. Care costs for catastrophic illnesses such as transplants, severe burns and injuries, or high-risk cardiovascular disease represent about 10\% of commercial health care expenses; in 1998 they exceeded $\$ 30$ billion. 'The National Cancer Institute estimates the direct medical costs of cancer to be $\$ 37$ billion. $^{2}$ The first-year cost of a liver transplant including immunosuppressants is $\$ 244,600$, for a heart transplant, $\$ 303,400 .{ }^{3}$ The cost of an immunosuppressant regimen ranges from $\$ 5,000-\$ 16,000$ per year, depending on the drugs and doses prescribed.

Immunosuppressant medications make prolonged graft survival possible, provided the medication is taken properly. For various and complicated reasons, however, $5 \%-45 \%$ of organ transplant recipients do not adhere to their medication regimens. ${ }^{4}$ Further, ignorance of complex drug interactions in this patient population can be devastating. Adding the cardiovascular agent diltiazem to a patient stabilized on cyclosporine can result in nephrotoxicity and graft loss. The cost of suboptimal management of an organ transplant recipient can be exhorbitant.

An ounce of prevention with specialized management can abort a pound of unforeseen calastrophic events and costs. A skilled specialty pharmacy can help reduce the cost of managing organ transplant patients by improving individual medication adherence, preventing drugrelated problems such as adverse drug interactions, and optimizing physician prescribing through targeted education.

Similarly, individuals living with HIV/AIDS represent a high, sometimes hidden, health care expense to managed care organizations. According to the World Health Organization, in 1999 the economic burden for prevention and treatment of HIV infection worldwide was approximately $\$ 14$ billion. Highly active antiretroviral drug "cocktail" regimens cost from $\$ 11,000-\$ 17,000$ per year. Frequent physician visits also contribute to the high cost of managing these patients. Many

Author

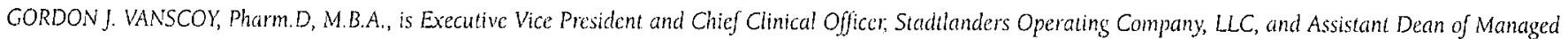
Carc and Associatc Professor; University of Pittsurgh School of Pharmacy, Pittsburgh, PA.

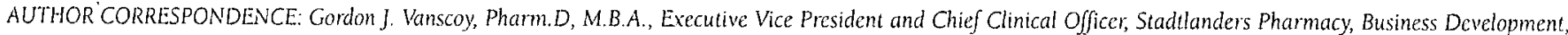
600 Penn Centcr Blvd., Pitlsburgh, PA 15235; Tel: 412-825-8350; Fax: 412-825-8397; E-mail; vanscoyg@@msx.upmc.edu. 
HIV/AIDS patients are prescribed a complex drug regimen of up to 500 pills per month. The unmonitored coadministration of the protease inhibitor ritonavir and the gastrointestinal motility agent cisapride can lead to cardiac arrhythmias and arrest. ${ }^{6}$ Missing even a few doses of a protease inhibitor may result in HIV virus mutation and resistance to that regimen and potentially other agents in an entire class of antiretroviral medications, adding to both the patient's risk and the cost to managed care companies. A single admission for treatment of an opportunistic infection may cost more than $\$ 13,000$.

While each catastrophic disease is unique, the medication therapies for these conditions present the same challenges to patients, payors, providers, and pharmacists: (1) the drugs are generally expensive; (2) the interactions within each drug regimen can be serious and must be continuously monitored; and (3) outcomes and overall costs are dramatically impacted if patients do not adhere to prescribed medication regimens.

"Specialty pharmacy enables managed care companies like ours to substantially restrain medical inflation, while improving patient care," said Rusty Hailey, vice president of specialty markets, Coventry Health Care. Specialty pharmacy creates a new role and a new industry focus for pharmacists. A team of skilled, specialized pharmacists with a large catastrophic patient population can benchmark and improve outcomes, manage overall costs for payors and patients, and practice pharmaceutical care at an advanced, population-based clinical level rarely found in other pharmacy carcers.

\section{- Critical Components of a Successful Specialty Pharmacy}

\section{Robust Clinical Expertise}

To develop and manage an effective specialty pharmacy, robust clinical expertise is essential. A multidisciplinary team with a core of highly educated and specialty-trained pharmacists is a key factor in a specialty pharmacy's ability to meas- ure and affect patient outcomes. Of even greater importance, however, is that the pharmacists are experts in the disease states in which they practice. A transplant pharmacist must have earned a reputation as the transplant medication expert among both patients and prescribers.

This starts with recruitment of inclividuals with specialty residency and fellowship experience. Next, a micro-to-macro practice transition occurs for these professionals, who now focus their approach to care on a population as a whole. Accomplishments (e.g., abstracts, presentations, data) are peer reviewed at national and international meetings. Finally, nothing beats the experience of working with thousands of special patients and hundreds of specialty providers.

Those delivering the program day-today must be competent in their specialty. A credible, curriculum-based, pharmacy staff development and credentialing process assures professional growth and ongoing competency. One method is to have staff return for an advanced degree and specialized residency training in the catastrophic illness of interest. However, most cannot afford the time and expense of a multiyear commitment away from the job. Another method is to partner with an academic institution to create an intense disease-specific educational sys$\mathrm{t} \mathrm{em}$ that goes far beyond a traditional continuing education program.

The University of Pittsburgh School of Pharmacy has partnered with Stadtlanders to create specialty credentialing programs in the advanced pharmaceutical management of HIV/AIDS, organ transplants, and serious mental illnesses. Randy Juhl, dean, University of Pittsburgh School of Pharmacy, said, "Each program joins mutual competencies and expertises to deliver 6-15 hours of intensive, curriculum-based training that requires participants to do considerable preparation, be actively involved in case discussions, and successfully complete a specialist examination."

All pharmacists who work on a specialty team should be credentialed every year. In addition, monthly continuing education sessions in the specialty should be provided to keep up with new advances. Even the pharmacist working in the quality-control area who checks medications before shipment must know the drugs, know the patients, and know the science, inside and out.

\section{A Catastrophic Medication Disease Management Program}

Disease management is still a relatively new concept in health care, particularly in the pharmaceutical industry. Thomas Bodenheimer defines disease management as "a systematic, population-based approach to identifying persons at risk, intervening with specific programs of care, and measuring clinical and other outcomes." Mainstream disease management programs do not exist for catastrophic cases such as organ transplants, HIV/AIDS, oncology, and hepatitis C.

The first step is to identify the goal of the program in terms of abilities to create change. The specialty pharmacy's goal is to optimize drug therapy benefits, minimize medication risks, and reduce overall health care costs through enhanced patient adherence and physician prescribing behaviors. Such a program provides the tools to affect outcomes in patients with catastrophic diseases.

Also necessary are a statistically robust base of patients and defined means to implement the programs costefficiently. All program components must be validated with leading physicians and health care industry authorities to ensure that protocols and methodologies are credible, reproducible, and scientifically based.

Benchmarking outcomes and costs in the patient population is next, followed by testing of a pilot program and full program implementation.

\section{Synergistic Disease Management Relationships with the Industry}

Pharmaceutical manufacturers of medications that are essential to optimal management of catastrophic illnesses can benefit from comparative market and 
outcomes data. In addition, manufacturers derive financial benefit from the ability of the specialty pharmacy to keep patients on preferred drugs through adherence programs and targeted physician prescribing education.

Specialty pharmacies can benefit in turn from the clinical expertise, data, and financial support of pharmaceutical manufacturers. Some pharmaceutical companies will underwrite an entire disease management program if a specialty pharmacy's patient population is large and if the pharmacy has a proven program to enable better use of the company's products. Financial support is important but must never violate the intent of the program to first be in the best interest of the patient and the health plan.

\section{A Statistically Significant Patient Population}

Only by managing the pharmaceutical care of a large patient population can a specialty pharmacy validate its data and protocols. The pharmacy is best positioned when it deals with thousands of disease-specific patients. Proving the efficacy of a disease management program necessitates that success can be replicated for a broad cross-section of a patient population. Results must be evaluated carefully, factoring out all mitigating variables.

\section{Effect on Physician Prescribing and Medication Adherence}

In addition to having a robust patient base, the pharmacy should deal with hundreds or even thousands of prescribers, many of whom are specialists, though primary care providers often benefit the most from the expertise that a specialty pharmacy can provide. A specialty pharmacist who practices in a clinical setting may be more aware than the prescribing physician of a broad array of drug interactions, adverse drug reactions, and evolving treatment protocols.

Prescriber interventions should not attempt to challenge authority. Instead, they can focus on encouraging informed decisions in a nonthreatening manner.
Disease-certified pharmacists can identify predetermined prescribing variances that likely will result in a negative clinical or financial outcome. Then they proceed through a defined decision-support process that prompts for selected data and makes clinical recommendations. Any written "intervention" to the prescriber must be nonthreatening; it must be patient- and problem-specific, provide evidence to support the recommended resolutions, provide a number of options, and, most of all, be simple and quick to read and complete.

Medication nonadherence varies depending on the timeframe, measurement method, and patient population. For the purposes of this article, a patient who misses enough doses or dose patterns to likely result in a negative clinical outcome is considered nonadherent. For example, missing eight or more doses of a protease inhibitor in a given month can dramatically increase the risk of HIV resistance.

Nonadherence occurs for a variety of reasons, including cost and unpleasant. side effects. Some patients skip doses when the symptoms of their condition disappear or when they feel healthy. Other patients just give up. A specialty pharmacy with skilled adherence counselors supported by master's-level social workers can help patients understand the importance of taking medication as prescribed.

No single method of augmenting adherence is foolproof. A combination of methods, depending on effectiveness, cost, and patient preference, is recommended: Educating patients on the importance of medication adherence is a great start. Refill surveillance and reminder programs will contribute to success. Regularly soliciting patient self-reporting of medication adherence and proactively intervening escalate success. Finally, risk-stratifying patients based on adherence and demographic risk factors enables targeted efforts.

\section{Managing Adverse Drug Reactions}

In the United States, more people die each year from adverse drug reactions than in automobile accidents." Estimates suggest that up to 1 in every 10 hospital admissions is due to the negative effect of a medication. Once in a hospital, up to 3 in every 10 patients experience an adverse medication reaction. Unfortunately, fewer than 1 in every 100 serious adverse drug reactions in hospitalized patients is reported. The cost of adverse drug reactions to society in terms of death, disability, and dollars is enormous. Most adverse drug reactions are preventable.

By using a systematic approach to detecting, preventing, and managing adverse drug reactions, a specialty pharmacist can help reduce their incidence. Although the U.S. Food and Drug Administration does not mandate that adverse drug reactions be reported, a specialty pharmacy should nevertheless report serious drug reactions and adverse trends via the MedWatch system. Specialty pharmacists must work in the best interest of the patient, the prescriber, and the payor to reduce mortality, morbidity, and the tremendous cost of adverse drug reactions. Patients who experience transient and clinically insignificant reactions can be counseled on how to manage the reaction and when the event will abate.

\section{Disease-Specific Education for Patients, Physicians, and Payors}

Because specialized pharmaceutical care is relatively new and because mainstream protocols change rapidly, patients, physicians, and payors will benefit from targeted education on how better to manage chronic high-risk conditions. Patients need broad as well as tailored information presented in a personal manner (e.g., live human contact, Internet interaction, literature, educational kits, articles, etc.). Physicians benefit from unbiased and timely evidence-based drug information that extends beyond the product labeling. Prescribers should have access to a Specialty Drug Informatics Center as a resource. Payors desire information about pharmacoeconomics, patient outcomes, formulary management, and prescribing 
trends. Overall, a specialty pharmacy has an important niche role in providing education about catastrophic disease medication and management.

\section{The Reimbursement Maze}

Medication reimbursement is a critical but challenging component of successfully managing catastrophic patient populations. Medication for chronic illnesses is expensive; prescription coverage is often limited. Most patients are unfamiliar with the types of coverage that are available to them or the limitations in their insurance policies. Because patients without adequate insurance coverage are more likely not to adhere to their medication regimens and are therefore at greater risk of a negative outcome, it is imperative that the specialty pharmacy help patients identify and secure third-party coverage for their medication.

An effective reimbursement professional must be knowledgeable about private and public programs throughout the country. The types and cost of coverage available for chronic and high-risk conditions varies dramatically among commercial insurance companies. Some customers may qualify for public assistance programs, including Medicare, Medicaid, and Medigap programs. Public programs are available for most catastrophic disease states as well.

\section{Implications for Managed Care Pharmacists}

\section{How Managed Care Deals with a Specialty Pharmacy}

All types of managed care organizations (MCOs) can use a specialty pharmacy to benefit their enrollees and support their prescribers, and for significant cost and quality management of a patient base that has not been a focus in the past. It is financially challenging for individual MCOs to devote the necessary skilled resources to have a measurable effect on catastrophic disease management. A national specialty pharmacy uses skilled professionals allocated across large, dis- ease-specific patient bases and many plans. How does an MCO use such a specialty pharmacy?

An MCO must recognize the need for such products and services. This requires research on the number of patients and costs of catastrophic diseases in their plan. Next, it must assess the credibility of a national specialty pharmacy. Flawless pharmaceutical distribution, a depth of reimbursement expertise, a proven disease management program, academically credentialed professionals, and experience in large populations of catastrophic illnesses are all necessary factors in the choice.

What does it cost? A desirable attribute for a specialty pharmacy is flexibility in financial relationships. Some MCOs and pharmacy benefits managers prefer a bundled program, including the cost of disease management in the medication reimbursement fee structure. Others unbundle the program and pay a fee for benchmarking their catastrophic population, using a cost-savings-sharing contract. Still others prefer a per member, per month disease management fee contract.

A critical aspect is getting plan members enrolled in the specialty pharmacy. One must consider the importance of member choice and the value of member pull-through. If a plan decides to offer voluntary enrollment, it is in its own best interests to encourage use of the specialty pharmacy. More restrictive plans require patients with prescriptions for catastrophic-disease medications to use the specialty pharmacy.

Overall, it is as simple as identifying the opportunity and partnering with a specialty pharmacy that will derive mutual benefit.

\section{A Unique Career Choice}

Specialty pharmacy provides an opporunity for pharmacists to apply cognitive skills to specific catastrophic illnesses. Pharmacists interested in this type of practice will:

- have a strong desire to combine clinical and economic skills;

- be very confident of their skills;
- be able to effectively deal with medical specialists;

- understand the principles of pharmacoepidemiology and outcomes management; and

- effectively communicate with patients, providers, payors, and pharmaceutical manulacturers.

As the success of specialty pharmacy flourishes, there is a growing demand for pharmacists in this field. In conjunction with a school of pharmacy, some specialty pharmacies offer advanced managed care residencies that prepare trainees to thrive in a specialty pharmacy or other managed care environment.

\section{The Future of Specialty Pharmacy}

\section{Expanding the Model To New Disease States}

A number of catastrophic conditions are ripe for specialty pharmacy programs. Such conditions include inflammatory bowel disease, the management of cancer with oral oncology agents, and muscular sclerosis. The criteria are relatively basic: the disease state or condition will be chronic, expensive to manage in terms of medications and other health care costs, and fraught with preventable or manageable drug-related problems.

An important consideration is to identify evolving trends in disease management. Ideally a disease state targeted for specialty pharmacy management would be one where there is tremendous potential for significant and immediate cost savings. Figure 1 graphs disease management program popularity and savings potential for the average health plan. Obviously, the top portion of the graph is financially desirable, while the left quadrants identify relatively untapped opportunities where there is little competition.

\section{Leveraging a Direct Delivery Model through the Internet}

The proliferation of health-related information on the Internet has empowered consumers. Patients now have an unprecedented ability to gather information about 


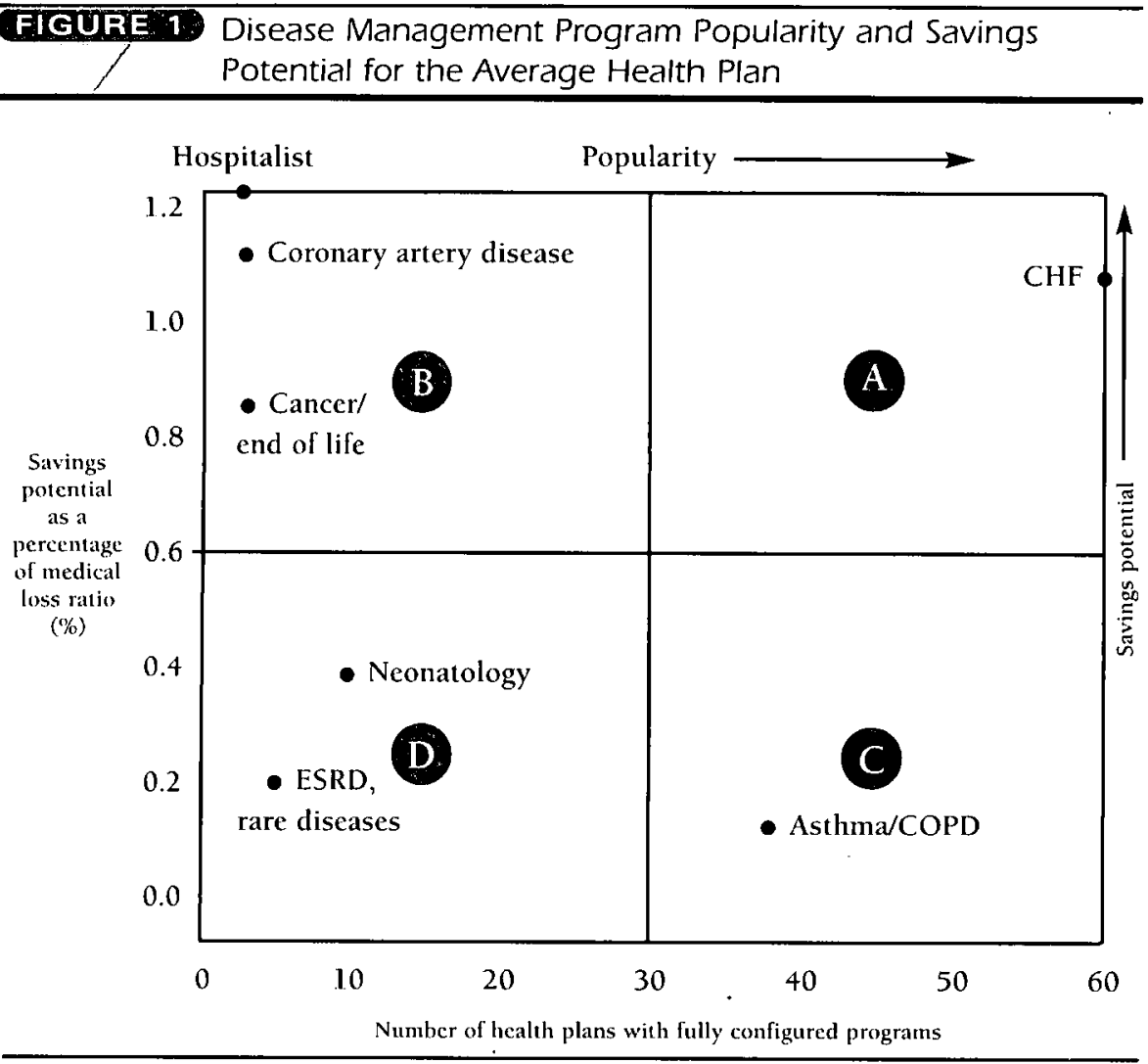

Notes: CHF is congestive heart failure. COPD is chronic obstructive pulmonary disease. ESRD is end-stage renal discase.

Source: Reprinted with permission from Decision Resources, Inc. Disease Management Consortium and Advisory Council LLC and Decision Resources, Inc., 1999.

their conditions and treatment options, as well as to order medications online.

Specialty pharmacies possess a modified mail-order distribution process with a multitude of insurance contractual relationships. Thus they have the necessary back-office capability on which to build an effective internet front end. A forwardthinking specialty pharmacy must create an effective Internet strategy that adds value for patients, payors, prescribers, and pharmaceutical manufacturers, or contemplate disintermediation. The synergies of combining the Internet with specialty pharmacy are immense, including order management efficiencies; using the enhance an MCO's capability to manage catastrophic illnesses. The value of providing specialized pharmaceutical care for patients with rare yet catastrophic conditions is enhancing care while managing costs.

Pharmacists thus have available a new career path that uses their ability to improve the quality of patient care in a unique managed care environment. There are many "niche" disease opportunities for specialty pharmacy on the horizon. Coupled with an effective Internet strategy, the future of this unique aspect of managed care pharmacy looks very promising.

\section{References}

1. Goodhue N. Managing limited resources: specialized programs curb calastrophic expenses and help avoid rationed care debate. Mngd Healthcare News 1999 (November): 41-43.

2. American Cancer Sociely. (1998). Cancer: basic facts. hup://www.cancer.org/statistics/cff98/basicfacts.html. Accessed January 2000.

3. Haubolde R. Cost implications of human organ and lissue transplantations, an update: 1999. Brookfield, WI: Milliman and Robertson, Inc., 1999.

4. Greenstein S, Seigel B. Compliance and noncompliance in patients with functioning rena! transplant: a multicenter study. Transplantation 1998; 66: 1718-26.

5. Fauci $A$. The AIDS epidemic: considerations for the 21.st century. New Eng J Med 1999; 34l (14): 1046-50.

6. Jansen Pharmaceuticals. Propulsid product information. Titusville, NJ: 1998.

7. Childress KJ, Saag M, Clougherty C, et al. Annual cost estimation and resource utilization for treating opportunistic illnesses associated with AIDS. Abstract N-23 from the 39th Interscience Conference on Antimicrobial Agents and Chemotherapy, San Francisco, CA, September 17-20, 1999.

8. Bodenheimer 'T. Disease management-promises and pitfalls. New Eng J Med 1999; 340 (15): 1202-05.

9. Lazarou J, Pomeranz BH, Corey PN. Incidence of adverse drug reactions in hospitalized paticnts: a meta-analysis of prospective studies. JAMA 1998 . 279: 1200-04. 